\title{
Identifying the Potential of Zero Charge and Faradaic Reactions by Step Potential Electrochemical Spectroscopy
}

\author{
$\underline{\text { Maksim Bahdanchyk }}^{1}$, Zahide Tuğba Sar1 ${ }^{1}$, and Antonello Vicenzo ${ }^{1}$ \\ ${ }^{1}$ Politecnico di Milano, Dipartimento di Chimica, Materiali Ingegneria Chimica "Giulio Natta" , via \\ Luigi Mancinelli 7, 20131 Milan, Italy \\ maksim.bahdanchyk@polimi.it
}

The study of electrode processes at carbon electrodes has great relevance to a variety of electrochemical applications. The wide range of carbon materials, their possible combination, the variability of electrode design and fabrication techniques, the specific application purposes, all these elements show the richness and complexity of this research area in electrochemistry. In particular, Activated Carbon (AC) electrodes are widely used for charge storage in supercapacitors and capacitive deionization cells. For their porous structure and surface characteristics, this class of electrodes may pose serious challenges to the capacity of routine electrochemical methods to achieve an in-depth analysis and the deconvolution of different electrode processes. A relatively new and interesting method, the Step Potential Electrochemical Spectroscopy (SPECS), has been shown to be an effective way to separate the contributions of faradaic and non-faradaic processes to the total current at AC electrodes ${ }^{1}$. The technique consists in applying to the working electrode (WE) a series of small potential steps and recording the current decay during a time interval long enough to allow for equilibration. Relatively simple models are fitted to the transients, to derive parameters of capacitive charging and faradaic processes. In the present work, we explore further the capability of SPECS to characterize electrode processes at AC electrodes, having in mind two objectives in particular: the determination of the Potential of Zero Charge $\left(E_{P Z C}\right)$ and the separation and possible identification of faradaic processes, through a closer examination of experimental data.

AC (YEC-8A) paste electrodes (80\% AC, 10\% carbon black and 10\% PTFE) on graphite current collector $\left(130 \mu \mathrm{m}\right.$ thick foil) were used as WE in $0.1 \mathrm{M} \mathrm{NaCl}_{\mathrm{aq}}$ electrolyte. The $E_{P Z C}$ was determined as the minimum of differential capacitance vs. potential plot (single-frequency EIS measurement at $5 \mathrm{mHz}$ ). SPECS was performed over the potential window from -0.25 to $0.75 \mathrm{~V}_{\text {SCE }}$ both in the anodic and cathodic direction, using a step of $25 \mathrm{mV}$ and equilibration time of $300 \mathrm{~s}$ (sampling time $0.1 \mathrm{~s}$ ). SPECS current transients are simulated by a two-exponential decay function with a residual current.

Normalized differential capacitance curves from EIS and SPECS are shown in Figure 1a. The value of $E_{P Z C}$ is found at about 0.175 and $0.190 \mathrm{~V}_{\mathrm{SCE}}$ by EIS and SPECS, respectively. Additionally, a good agreement is found between the absolute capacitance minimum determined by either technique at electrolyte concentration from 0.01 to $1.0 \mathrm{M}$, see inset of Figure 1a. The plot of residual currents vs. potential from the analysis of SPECS data is shown in Figure 1b. The trend of the currents, in both scan directions, highlights two "inversion" potentials: at about $0 \mathrm{~V}_{\mathrm{SCE}}$, during forward polarization, and at about $0.57 \mathrm{~V}_{\mathrm{SCE}}$, during backward polarization; in other words, the residual current is positive on the anodic side and negative on the cathodic side vs. the inversion potential, thus recreating the typical shape of a polarization curve. With this premise, though speculative, we identify tentatively two different reactions in the anodic and in the cathodic scan of the SPECS measurements, corresponding to carbon oxidation and oxygen reduction, respectively. Notably, the equilibrium potential of these reactions is reasonably close to the inversion potential observed in Figure $1 \mathrm{~b}$.
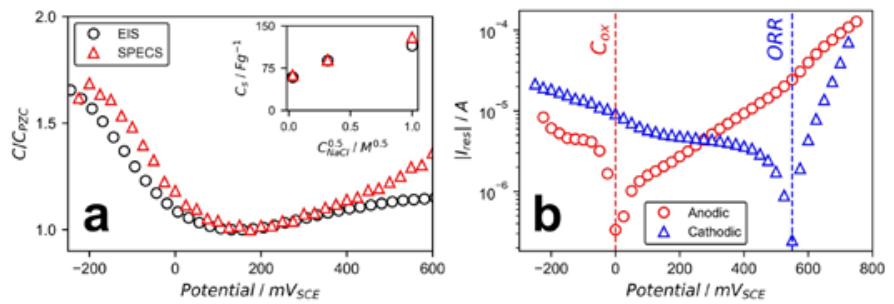

Figure 1 - a) Differential capacitance and b) residual currents vs. potential, obtained from the SPECS current transients during a forward and backward scan

1.Dupont, M.F. \& Donne, S.W. Separating Faradaic and Non-Faradaic Charge Storage Contributions in Activated Carbon Electrochemical Capacitors Using Electrochemical Methods I . Step Potential Electrochemical Spectroscopy. 162, 1246-1254 (2015). 